\title{
Zur Photooxydation der Aldehydgruppe: I. Terephtalaldehyd
}

\author{
(II. Mitteilung über chemische Lichtwirkungen) ${ }^{1}$
}

von

Hermann Suida.

Aus dem photochemischen Laboratorium der $k$. $k$. graphischen Leln- und Versuchsanstalt in Wien.

(Mit 4 Textfiguren.)

(Vorgelegt in der Sitzung am 20. Juni 1912.)

Das Licht ist nicht nur imstande, oxydierende Eigenschaften von Verbindungen hervorzurufen oder $z u$ verstärken, sondern auch den Luftsauerstoff in ein energisches Oxydationsmittel umzuwandehn. Diese spezifische katalytische Wirkung des Lichtes ist frühzeitig erkannt, die Vorgänge selbst sind aber noch wenig studiert worden.

Engler und Weißberg" haben in ihrer zusammenfassenden Studie über Autoxydationsvorgänge einzelne Beobachtungen über die Förderung der Autoxydation durch Lichtenergie angeführt. K. Gebhar $\mathrm{d}^{3}$ hat durch seine Anschauungen über die Verteilung der Valenzkräfte in organischen Verbindungen und ihre Beeinflussung durch Lichtstrahlen ein weites Feld für neue Untersuchungen der Teilvorgänge bei chemischen und photochemischen Reaktionen eröffnet. Zuletzt haben

1 Vgl. I. Mitt. Uiber diesen Gegenstand. Journ. f. prakt. Chem., 84 (N. F.) S.7-7(1911).

$2 \rightarrow$ Kritische Studien iiber die Vorgänge der Autoxydation. Braunschweig 1904.

s Journ. f. prakt. Chem., 84 (A. H.), 561 (1911). 
Ciamician und Silber Angaben über Autoxydation von gesättigten Kohlenwasserstoffen im Lichte gemacht.

Unzweifelhaft sind die Peroxyde als labile $Z$ wischenglieder zwischen zwei stabilen Oxydationsstufen anzusehen.

Der eigentlichen Oxydation geht immer eine Anlagerung* von Sauerstoff roraus, wodurch der Sauerstoff eine gesteigerte Aktivität erhält; dieses Anlagerungsprodukt, das Peroxyd, kann dann unter Herbeiziehung eines zweiten unveränderten Moleküls zur nächsthöheren, stabilen Oxydationsstufe übergehen, die keinen aktiven Sauerstoff mehr enthält, wieder anlagerlingsfähig sein kann, aber ein ganz neues Individuum mit oft grundverschiedenen Eigenschaften darstellt. Die Gleichungen

$$
\begin{aligned}
\mathrm{CX}+\mathrm{O}_{2} & \rightarrow \mathrm{CXO} \\
\mathrm{CXO}+\mathrm{C} X & \rightarrow 2 \mathrm{CXO}
\end{aligned}
$$

würden diesen Vorgang veranschaulichen. Das Peroxyd $\mathrm{CXO}_{3}$ ist imstande, an einen geeigneten Akzeptor (im Sinne Engler's) ein Atom Sauerstoff abzugeben.

Wesentlich ist bei der Photoautoxydation, daß der zu oxydierende Körper befähigt sein muß, selbst durch Lichtzufuhr anlagerungsfähig $z u$ werden (d. h. freie Valenz $z u$ äußern).

Es ist anzunehmen, daß die einzelnen Glieder der Reihe wohldefinierter Substanzen vom Kohlenwasserstoff bis zur Säure je nach dem Bau ihrer charakteristischen Gruppe eine Oxydationsfähigkeit in verschiedenem Grade besitzen. Von der Aldehydgruppe ist ohneweiters eine viel raschere und leichtere Oxydierbarkeit zu erwarten. Auch der Umstand, daß Ciamician und Silber bei der Oxydation von Toluol und Xylolen in Gegenwart von Wasser am Lichte Aldehyde nur in geringer Menge, oft aber gar nicht vorfanden, während reichliche Mengen Karbonsäuren und Kohlenwasserstoff vorhanden waren, läßt schließen, daß die Aldehydphase infolge ihrer raschen weiteren Oxydation sich der Beobachtung oft entzieht.

1 Ber. d. Deutsch. chem. Ges., 45, 38 (1912).

$\because$ Vgl. J. Schmidlin und R. Lang, . Molekülverbindungen als erste Reaktionsstufen *. Chem. Zentralbl., 1912 (1), 1769. 
Dementsprechend müßte die Peroxydbildung eine sehr rasche sein, üm beobachtet werden zu können.

Einer Täuschung zu entgehen, habe ich Benzoësäure, Benzaldehyd. und Toluol äquimolekular in Benzol gelöst, vor der Quarzlampe einen Tag belichtet und die Stärke der Peroxydbildung geprüft. Während Toluol und Benzaldehyd erhebliche Mengen Peroxyd (gemessen durch die Jodausscheidung aus saurer Jodkaliumlösung) erkennen ließen, war bei der Benzoësäure keines nachweisbar.

Hierbei zeigte sich aber, daß Benzaldehyd, der frisch in Benzol gelöst wurde, auch ohne vorhergehende Belichtung erheblich stärkere Peroxydbildung zeigte als Toluol, daß die Dunkelreaktion der Aldehydgruppe eine bedeutend raschere ist als die der Methylgruppe.

Hierbei läßt sich freilich nicht entscheiden, ob dies von einem erheblichen Peroxydgehalt des Benzaldehyds an sich oder von einer rascheren Oxydation beim Schütteln in Benzol mit saurer Jodkaliumlösung und Luft herrührt. Die letztere Annahme besitzt größere Wahrscheinlichkeit, indem hier der Aldehyd als Autoxydator, Jodwasserstoff als Akzeptor wirkt.

\section{Autoxydation von Terephtalaldehyd im Lichte.}

Terephtalaldehyd ist in fester Form sehr beständig und auch in Lösung hält er sich unverändert. Ich habe seine gesättigte Lösung in Benzol in Berührung mit Luft durch einen Monat im Dunkeln aufbewahrt, ohne daß eine sichtbare Veränderung eingetreten wäre. Auch fünfstündiges Kochen am Rückflußkühler unter Lichtabschluß ergab keinerlei Reaktion.

Vorversuche vor derQuecksilberdampf-Quarzlampe zeigten, daß sich nach wenigen Stunden an der Lichtseite der Eprouvette eine weiße Substanz krystallinisch ausgeschieden hatte.

Eine Belichtung von $4 \mathrm{~g}$ Terephtalaldehyd in $50 \mathrm{~cm}^{3}$ heißem Benzol lieferte nach $5 \frac{1}{2}$ Stunden $1 \cdot 1 \mathrm{~g}$, in Benzol unlösliche, krystallinische, farblose Substanz.

Das verdampfte Benzol wurde von Zeit zu Zeit ersetzt; die Reaktionstemperatur lag bei $70^{\circ}$.

Ein zweiter Versuch wurde derart ausgeführt, daß drei Eprouvetten mit einer Lösung von Terephtalaldehyd in Benzol 
in einem Kühtgefüb, das gegen die Lampe ein Glasfenster besa $\beta$ und durch das ein Wasserstrom von 6 bis $8^{\circ}$ zirkulierte, belichtet wurden. Hier trat die Reaktion ungefähr sechsmal so langsam ein. Die Lichtschwächung durch die dünne Glasplatte und die einige Millimeter dicke Wasserschicht kann nicht allzu erheblich die Reaktionsgeschwindigkeit verringern, wohl aber ist es die höhere Temperatur, welche die Reaktionsgeschwindigkeit erheblich vergrößert.

Die abgeschiedene Substanz wurde jeweilig abgesaugt und mit kaltem Benzol gewaschen.

Die Benzollösung gibt mit saurer Jodkaliumlösung Peroxydreaktion, nicht aber die ausgeschiedene Substanz.

Hierbei zeigt sicl eine gewisse Acidität der Benzollösung. die dem Anteil an gclöstem Reaktionsprodukt entspricht, der im Verhältnis zum Ausgeschiedenen aber sehr gering ist.

So verbrauchten je $10 \mathrm{~cm}^{3}$ der abfiltrierten Lösung bui direkter Titration mit Kalilauge und Phenolphtalein (unter Schütteln), beziehungsweisc nach dem zwölfstündigen Schütteln mit saurer Jodkaliumlösung $7 \cdot 33 \mathrm{~cm}^{3} n / 25 \mathrm{KOH}$, beziehungsweise $2 \cdot 10 \mathrm{~cm}^{3}{ }^{n} / 25$ Thiosulfatlösung.

Eine zugleich ausgeführte Titration mit je $10 \mathrm{~cm}^{3}$ unbelichteter Terephtalaldehydlösung ergab einen Verbrauch von $1.02 \mathrm{~cm}^{*}{ }^{n / 25} \mathrm{KOH}$, beziehungsweise $0.9 \mathrm{~cm}^{3}{ }^{n} / 25$ Thiosulfatlösung.

Bei einem anderen Versuche zeigte sich, daß durch füntstündiges Kochen der belichteten, abfiltrierten Lösung unter Lichtabschluß die Acidität um einen geringen Betrag zugenommen hatte, während zugleich der Peroxydgehalt eine deutliche Abnahme zeigte.

Obwohl die Titrationen sehr genaue Resultate liefern. lassen die geringen Differenzen keinen einwandfreien SchluB zu und können nur die Richtung bezeichnen, nach der sich die Reaktion ändert.

Je $10 \mathrm{~cm}^{3}$ einer belichteten, filtrierten Terephtalaldehydlösung in Benzol verbrauchten vor dem Kochen $2.44 \mathrm{~cm}$;

1 Bestimmung des aktiven Sauerstoffes nach Baeyer, Ber. d. Deutsch. chem. Ges., 34, 740 (1901); vergl. auch Gebhard, Zeitschr. f. angew. Chemie. $23,820(1910)$. 
$n / 25 \mathrm{KOH}$, beziehungsweise $0.85 \mathrm{~cm}^{2} n / 25$ Thiosulfat, nach dem Kochen $2.56 \mathrm{~cm}^{3} \mathrm{n} / 25 \mathrm{KOH}$, beziehungsweise $0.50 \mathrm{~cm}^{3}$ $n / 25$ Thiosulfat, während eine zugleich ausgeführte Blindprobe einen Verbrauch von $1.05 \mathrm{~cm}^{5} n / 25 \mathrm{KOH}$, beziehungsweise $0.50 \mathrm{~cm}^{3} n / 25$ Thiosulfat ergab.

Das Peroxyd hält sich hier jedenfalls nur kurze Zeit und wird zugunsten der Süurebildung zerlegt.

Die ausgeschiedene Substanz besteht aus baumartig verzweigten Krystallaggregaten, die aber ein Gemenge von Terephtalaldehydsäure und Terephtalsäure vorstellen. Die Annahme, daß ein Kondensationsprodukt oder eine Peroxydphtalsäure ${ }^{1}$ ausgeschieden werde, traf nicht $z u$.

$0.2679 g$ bei $100^{\circ}$ getrockneter Substanz verbrauchten bei der Titration mit $n / 2$, Kalilauge unter Verwendung von Phenolphtalein $0.1070 \mathrm{~g} \mathrm{KOH}$, woraus sich für eine einbasische Säure ein Molekulargewicht von $140 \cdot 3$ ergibt; demgegenüber hat Terephtalaldehydsäure das Molekulargewicht $150 \cdot 05$, Terephtalsäure $166 \cdot 05$.

Die rohe Säure wurde durch Lösen in Soda, Ausschütteln mit Äther und Fällen mit Salzsäure gereinigt. Ein geeignetes Lösungsmittel zum Umkrystallisieren konnte nicht gefunden werden. Sie zeigte so bei $195^{\circ}$ ein schwaches Sintern, blieb weiterhin bis $300^{\circ}$ unverändert; auch Einbringen in ein auf $250^{\circ}$ vorgewärmtes Bad bewirkte nur ein etwas stärkeres Sintern, aber kein Schmelzen. Die Säure gibt in alkoholischer Lösung ein Phenylhydrazon, enthält also eine Karbonylgruppe, aber dieses Phenylhydrazon konnte auch nicht befriedigend gereinigt werden; es begann sich stets um $245^{\circ}$ zu zersetzen, ergab aber mit reinem, bei $226^{\circ}$ schmelzendem Terephtalaldehydsäurephenylhydrazon keine wesentliche Schmelzpunkterniedrigung.

Ein Teil der Rohsäuren wurde über das Kalisalz ins Silbersalz übergeführt, das ziemlich unbeständig war und mit Alkohol getrocknet wurde. Die hornartige Masse wurde pulverisiert und mit überschüssigem Jodmethyl zwei Tage im verschlossenen Gefäß digeriert; der Reaktionsmasse konnte mit

1 Vergl. Baeyer, Ber. d. Deutsch. chem. Ges., 34, 766 (1901). 
Benzol ein krystallisierter Ester entzogen werden, der aber trotz wiederholtem Umlösen aus Petroläther ganz undeutliche Schmelzpunkte zwischen 50 bis $95^{\circ}$ zeigte, also wieder ein Gemisch war; endlich konnte durch fraktioniertes Umkrystallisieren aus heißem Wasser ein Anteil in Nadeln krystallisiert erhalten werden, der einen scharfen Schmelzpunkt von 140 bis $141^{\circ}$ zeigte und durch den Mischschmeizpunkt mit einem bei 141 bis $142^{\circ}$ schmelzenden Präparat von Terephtalsäuredimethylester sich mit diesem als identisch erwies; auch zeigte dieser Teil keine Phenylhydrazonbildung, während der rohe Ester in methylalkoholischer Lösung ein Phenylhydrazon gab, das aber nicht rein zu erhalten war.

Bezüglich der Eigenschaften der Terephtalaldehydsäure und ihrer Derivate verweise ich auf eine kürzlich publizierte Abhandlung von $R$. Wegscheider und $H$. Suida. ${ }^{1}$

Ich habe gefunden, daß durch mehrmaliges Verreiben eines Gemisches von Terephtalsäure und Terephtalaldehydsäure und ebenso ihrer neutralen Methylester mit käuflicher Bisulfitlauge diese Körper quantitativ getrennt werden können, welche Methode viel rascher und bequemer ausführbar ist als die früher angewandte Heißextraktion der Säuregemische mit Chloroform. ${ }^{2}$

Auf diese Weise konnte ich auch das Säuregemisch der Lichtreaktion und ebenso das aus den Silbersaizen gewonnene Estergemisch trennen.

Die Esterbisulfitverbindung und ebenso die Bisulfitverbindung der Säure wird erst durch Kochen mit verdünnter Schwefelsäure zerlegt, der freie Ester und die Säure krystallisieren aus der wässerigen, sauren Lösung aus. Bei dieser Behandlung des Esters wird aber ein Teil verseift und es ist nötig, durch Übersättigen mit Ammoniak und Ausschütteln mit Äther den Ester zu reinigen.

1 Sitzungsberichte der kais. Akademie der Wissenschaften in Wien, mathem.-naturw. Klasse, Abt. IIb, 7. Juni 1912.

2 Diese Methode wurde von Wegs cheider und Suida (1. c.) verwendet und ist auch in der kürzlich erschienenen Ablandlung von Simonis (Ber. d. Deutsch. chem. Ges., 45, 1588 [1912]) genannt. 
Der so erhaltene Ester schmilzt bei 58 bis $60^{\circ}$ und zeigt mit reinem Terephtalaldehydsäureester (vom Schmelzpunkt $63^{\circ}$ ) keine Erniedrigung. Zur weiteren Identifizierung wurde ein Teil in das Phenylhydrazon übergeführt, welches sich mit dem hei 144 bis $146^{\circ}$ schmelzenden Terephtalaldehydsäuremethylesterphenylhydrazon als identisch erwies. Der Ester reduziert ammoniakalische Silberlösung in der Wärme unter Spiegelbildung.

Der in Bisulfit unlösliche Anteil des Estergemisches gibt kein Phenylhydrazon und schmilzt nach einmaligem Umlösen aus Petroläther bei 137 bis $138^{\circ}$; durch den Mischschmelzpunkt mit einem bei 140 bis $141^{\circ} \mathrm{schmelzenden} \mathrm{Präparat} \mathrm{von}$ reinem Terephtalsäuredimethylester konnte der Ester als mit diesem identisch erkannt werden; dieser Ester reduziert ammoniakalische Silberlösung nicht.

Von den durch Bisulfit getrennten rohen Säuren lieferte die bisulfitlösliche ein bei 217 bis $219^{\circ}$ schmelzendes Phenylhydrazon, das mit einem bei 225 bis $226^{\circ}$ schmelzenden Präparat von reinem Terephtalaldehydsäurephenylhydrazon keine Schmelzpunkterniedrigung lieferte. Die in Bisulfit unlösliche Säure gibt kein Phenylhydrazon.

Hieraus zeigt sich, daß bei der Autoxydation des Terephtalaldehyds im Lichte ein Gemisch von Terephtalsäure und Terephtalaldehydsäure entsteht; ungefähr zwei Drittel des Gemisches bestehen aus Aldehydsäure, welche Menge auch bei der Oxydation des Terephtalaldehyds mit der berechneten Menge Kaliumbichromat erübrigt.

Diese Lichtreaktion verläuft am besten im Sonnenlichte; aber auch diffuses Tageslicht im Zimmer genügt, um schon nach wenigen Stunden die Ausscheidung der Säuren zu bewirken. Der nötige Sauerstoff wird nur durch die verhältnismäßig kleine, zirka $1 \mathrm{~cm}^{2}$ große Flüssigkeitsoberfäche durch Diffusion zugeführt.

Alle Versuche wurden in kalibrierten Glaseprouvetten aus gleichem weißen Glase von gleicher innerer und äußerer Lichte und Wandstärke $(0.7 \mathrm{~mm})$ durchgeführt, so daß bei 
gleichartige ${ }^{\circ}$ Bestrahlung ein quantitativer Vergleich z.ulässig ist.

Die Reaktion wird so wie bei der Autoxydation des Benzaldehyds nur durch den zugeführten Sauerstoff bewirkt, wobei das Licht eine äußerst heftig beschleunigende Wirkung ausübt: sie kommt nicht durch gleichzeitige innere Reduktion und Oxydation zustande, etwa wie die von Pribram und Franke ${ }^{1}$ beschriebene Bildung von Ameisensäure aus Formaldehyd. Beweisend hiefür ist ein Versuch, bei dem Terephtalaldehyd in Benzollösung unter Durchleiten eines über reduziertes, glühendes Kupfer geführten, langsamen Kohlensäurestromes viele Stunden belichtet wurde, ohne daßs eine Ausscheidung eintrat.

Ein weiterer Versuch ging dahin aus, festzustellen, ob die Reaktion bei intensiver Sauerstoffzufuhr auch im Dunkeln eine meßbare Geschwindigkeit erlange. Zu diesem $Z$ wecke wurden drei Eprouvetten mit je $10 \mathrm{~cm}^{2}$ Terephtalaldehydlösung; ron denen eine mit Stanniol umwickelt und so vor Lichtzufuhr vollständig geschützt war, nebeneinander dem Sonnenlichte ausgesetzt. Von den zwei nicht abgeblendeten Röhren war die eine mit Watte verschlossen, in die zweite wurde ebenso wie in die abgeblendete durch ein Glasrohr, das bis zum Boden reichte, ein langsamer Luftstrom eingeleitet. Von Zeit zu Zeit mußten die Glasrohre ausgeputzt werden, da sie sich bald mit ausgeschiedener Substanz verlegten.

Die Belichtung dauerte bei einer Lufttemperatur von 20 bis $23^{\circ}$ vier Stunden. Die ausgeschiedene Menge an Säuregemisch betrug in der ersten Eprouvette, durch die kein Luftstrom geführt worden war, $0.025 g(10 \%$ des vorhandenen Aldehyds), in der zweiten, durch die ein Luftstrom geführt worden war, $0.068 g(26 \cdot 1 \%$ des vorhandenen Aidehyds). In der abgeblendeten Röhre war keine Säure abgeschieden worden; der Pfropf, der sich stets an der Mündung des Einleitungsrohres bildete, bestand nur aus Terephtalaldehyd, der sich bei der starken örtlichen Verdampfung des Benzols aus

1 Sitzungsherichte der kais. Akademie der Wissenschaften in Wien, mathem,-naturw. Klasse, Abt. IIb, Februar 1912. 
der fast gesättigten Lösung ausscheidet. Beim Stehenlassen der Lösung nach Abstellung des Luftstromes löste er sich von selbst wieder auf.

In einem weiteren Versuche wurde die Oxydationsgeschwindigkeit des Terephtalaldehyds mit der des Benzaldehyds am Lichte in Vergleich gestellt. Zugleich wurde untersucht, ob ein geringer Zusatz von Nitrobenzol ebenso wie bei der Autoxydation des Xylols ${ }^{1}$ einen Einfluß ausübt.

Zwei Eprouvetten mit einer Lösung von je $0 \cdot 2 g$ Terephtalaldehyd in $10 \mathrm{~cm}^{3}$ Benzol, von welchen zu einer der Lösungen zwei Tropfen Nitrobenzol zugesetzt war, und eine Eprouvette mit der äquimolekularen Menge von $0.16 \mathrm{~g}$ Benzaldehyd in $10 \mathrm{~cm}^{3}$ Benzol wurden zugleich sechs Stunden an der Sonne und einen Tag im diffusen Licht exponiert. Gleichzeitig wurden je $10 \mathrm{~cm}^{3}$ der Terephtalaldehyd- sowie der Benzaldehydlösung bei gleicher Dauer im Dunkeln der Luft ausgesetzt. Alle Röhren waren mit Baumwolle lose verschlossen. Hernach wurden alle Lösungen mit je $20 \mathrm{~cm}^{3}$ Wasser und $20 \mathrm{~cm}^{3}$ Benzol in Kolben gespült und unter starkem Schütteln mit ${ }^{n / 25}$ Kalilauge und Phenolphtalein titriert, dann zu jeder Mischung $20 \mathrm{~cm}^{3}$ Jodkaliumlösung (20 $\mathrm{g}$ in $600 \mathrm{~cm}^{2}$ Wasser) und $4 \mathrm{~cm}^{3}$ verdünnter Schwefelsäure zugesetzt und verschlossen digeriert, hiervon sieben Stunden geschüttelt. Dann wurde mit ${ }^{n / 25}$ Thiosulfat die Jodausscheidung gemessen.

Es verbrauchten: $\quad n_{25,5}$ Kaliliauge ${ }^{n}$.25 Thiosulfatlösung Terephtalaldehyd unbelichtet ... $1.25 \mathrm{~cm}^{3} \quad 1.31 \mathrm{~cm}^{3}$ Terephtalaldehyd belichtet ....14.130 $1 \cdot 64$

Terephtalaldehyd + Nitrobenzol

belichtet ........... $12 \cdot 50 \quad 1 \cdot 83$

Benzaldehyd unbelichtet .....6.6. $6 \cdot 20 \quad 1 \cdot 10$

Benzaldehyd belichtet .......17.25 $4 \cdot 45$

Differenz zwischen Licht-

und Dunkelreaktion für

Terephtalaldehyd..........13.35 0.33

Benzaldehyd............11 $05 \quad 3 \cdot 35$

1 Ujber die Autoxydation von Benzolhomologen im Lichte und ihre Beeinflussung durch Gegenwart von Nitrobenzol berichte ich in einer binnen kurzem folgenden Abhandlung. 
Nimmt man an, daß von einer Aldehydgruppe ein Molekül Sauerstoff gebunden wird, $-\mathrm{CHO}+\mathrm{O}_{2}=-\mathrm{CHO}_{3}$, und daß eine so veränderte Aldehydgruppe mit einer zweiten unveränderten unter Bildung von Karboxylgruppen reagiert, $\mathrm{CHO}_{3}+-\mathrm{CHO}=2-\mathrm{COOH}$, womit nur eine Bruttogleichung gegeben sein soll, und geht man von der bei den Persäuren gefundenen Tatsache aus, daß ein Peroxyd an Jodwasserstoff ein Atom Sauerstoff abgibt, so kann man den Verbrauch an Thiosulfat auch in äquivalenten Mengen Kalilauge ausdrücken, woraus sich ergeben würde, daß die Sauerstoffaufnahme ron Terephtalaldehyd und Benzaldehyd durch Lichtzufuhr sich wie 13.68: 14. 40 verhält, also nahezu gleich ist. Zieht man aber in Betracht, daß aus Terephtalaldehyd etwa ein Drittel Terephtalsäure und zwei Drittel Terephtalaldehydsäure entsteht, so erscheint es als nicht unwahrscheinlich anzunehmen, daß die Oxydationsgeschwindigkeit der Aldehydgruppe durch eine zweite paraständige gleiche Gruppe ivesentlich gehemmt wird. falls durch Diffusion grenügend überschüssiger Sauerstoff geliefert wird.

Die Sekundärreaktion verläuft beim Terephtalaldehyd rascher als beim Benzaldehyd, weshalb ein höherer Peroxydgehalt des Gemisches beim Benzaldehyd vorliegt. Die Ursache hiefür findet darin eine befriedigende Erklärung, daß beim Terephtalaldehyd das in Benzol fast unlösliche Reaktionsprodukt dem System sofort entzogen wird, weshalb sich das Gleichgewicht zwischen Peroxyd und Säuren zugunsten der letzteren verschiebt.

Nitrobenzol wirkt hier nicht beschleunigend wie bei der Methylgruppe (a. a. O.), sondern behindernd und man kann an Nebenreaktionen oder, falls Terephtalaldehyd und Nitrobenzol keinerlei Molekülverbindung ${ }^{1}$ einzugehen vermögen, an eine optische Schirmwirkung denken.

Diese Oxydationen sind als typische Lichtreaktionen anzusehen, bei welchen das Licht als Reaktionsbeschleuniger einer sehr langsam verlaufenden Dunkelreaktion wirkt.

1 Vergl. J. Schmidin und R. L,ang, 1. e. 
Die Rolle des Peroxyds als Reaktionszwischenstufe erfordert eingehendes Studium und ich behalte mir diesbezüglich weitere Mitteilungen vor.

Um festzustellen, durch welche Strahlengattung die Reaktion besonders begünstigt wird, habe ich je $10 \mathrm{~cm}^{3}$ der Terephtalaldehydlösung in gleichen Glaseprouvetten nebeneinander hinter $1 \mathrm{~cm}$ dicken, von zwei Quarzplatten eingeschlossenen Schichten von Wasser, vierprozentiger wässeriger Chininsulfatlösung und vierprozentiger wässeriger Kaliummonochromatlösung im direkten Sonnenlicht unter sorgfältiger Abhaltung jeglichen Seitenlichtes exponiert. Die Lufttemperatur: betrug im Mittel $15^{\circ} \mathrm{C}$. Die ausgeschiedenen Mengen von Säuren wurden nach zehnstündiger Belichtung gesammelt und betrugen hinter Wasser 105, hinter Chininsulfat 5 , hinter Kaliummonochromat $2 \mathrm{mg}$. Diese Zahlen machen keinen Anspruch auf volle Genauigkeit, sind aber der Größenordnung nach jedenfalls vergleichbar.

Wiederholte Versuche bei verschiedener Lichtstärle zeigten, daß hinter Chininsulfat und Monochromat die Reaktion stets merkbar, aber sehr gering, eintrat, die Ausbeute hinter Monochromat aber gegen der hinter Chininsulfat stets zurückblieb.

Vierprozentige Kaliummonochromatiösung läßt nach Ede $\mathrm{r}^{2}$ nur rote und gelbe Strahlen bis etwa $\lambda=480 \mu \mu$, wässerige vierprozentige Chininsulfatlösung nach eigenen Versuchen bis $\lambda=403 \mu \mu$ durch; es wird durch dieses letztere also das gesamte Ultraviolett ausgeschaltet; hievon habe ich mich durch Herstellung einer beiläufigen Absorptionskurve überzeugt.

$\mathrm{Zu}$ diesem $Z$ wecke habe ich eine $11 \mathrm{~mm}$ dicke, zwischen Quarzplatten eingeschlossene Schichte der Lösung vor den Spalt eines Quarzspektrographen angebracht und beim Lichte eines zwischen Tantalstiften überschlagenden Funkens eine photographische Platte 5,10,20,40,80, 160,320,640 Sekunden belichtet. Diese wie alle anderen Aufnahmen wurden mit dem

1 »Beiträige zur Photoshemie und Spektralanalyser, Wien 1904, II, 127. 
Sleichen, stets frisch bereiteten Pyrogallolsoda-Entwickler sieben Minuten entwickelt. Die gegen Ultraviolett vorschreitenden Enden der Schwärzungen geben ins Zeit-Wellenlängendiagramm übertragen eine kontinuierliche Kurve von folgender Gestaltung.

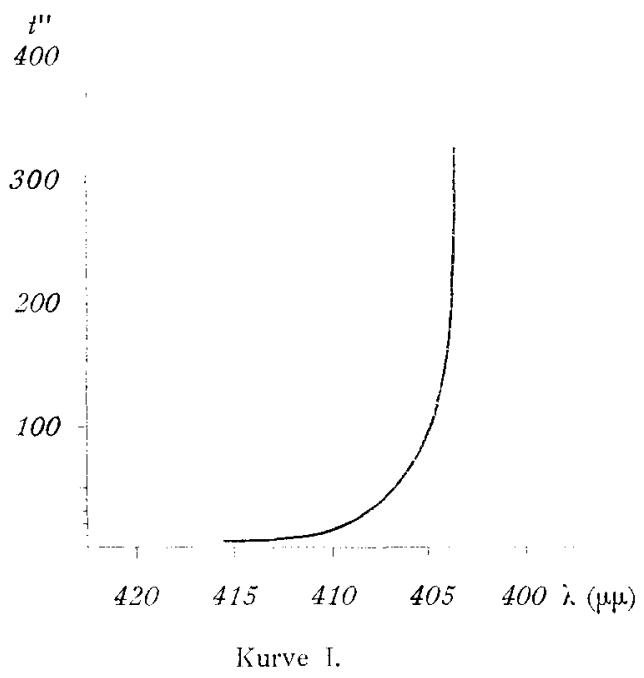

Auf Vorschlag von Herm Hofrat J. M. Eder wurde der Tantalfunke verwendet; sein ungemein linienreiches, bis ins äußerste Ultraviolett geschlossene Spektrum ${ }^{1}$ ist bei kürzester Belichtungszeit lichtstark genug, um bis gegen $200 \mu \mu$ auf der Platte einen deutlichen Eindruck hervorzurufen, weshalb es sich zur Bestimmung der Absorption im Ultravioletten besonders eignet. Die Absorptionskurve bei Verwendung von Sonnenlicht zeigt dieselbe Gestaltung.

Hieraus erhellt, daß die Autoxydation des Terephtalaldehyds fast nur durch das ultraviolette Licht bewirkt wird, die sogenannten "aktinischen " blauen und violetten Strahlen sich an der Reaktion nur zum geringsten beteiligen, daß aber auch langwelliges, rotes und gelbes Licht die Reaktion, wenn auch sehr schwach hervorruft.

1 Vergl. Eder und Valenta, Atlas der typischen Metallspektren «, Wien 1911. 
Das verwendete Sonnenlicht enthält ultraviolette Strahlen bis zu,zirka 300 p

Hierbei ist allerdings das Eigenabsorptionsvermögen des Terephtalaldehyds, eventuell auch seines Lösungsmittels ins Auge zu fassen.

Über die Absorption des Benzols liegt eine Reihe ron Messungen vor. Aus H. Kayser's »Handbuch der Spektroskopie «, 1905, III. Bd., p. 483 bis 485 läßt sich entnehmen, daß starke Absorption im Ultrarot, je ein sehr schmales Band im Rot, Orange und Grün und endlich selektive Absorption im Ultravioletten von $270 \mu \mu$ abwärts vorhanden ist. Für die bei der vorliegenden Reaktion in Betracht kommenden Strahlen (etwa 400 bis 300 s. ist das Lösungsmittel Benzol also durchlässig.

Die Absorption des Terephtalaldehyds habe ich qualitativ unter Benutzung des von $\mathrm{Eder}$ vorgeschlagenen Tantalfunkens spektrographisch bestimmt.

$\mathrm{Zu}$ diesem $\mathrm{Zwecke}$ habe ich die beiläufigen Absorptionskurven der $11 \mathrm{~mm}$ dicken, zwischen Quarzplatten eingeschlossenen Schichten von $2,1,1 / 2,1 / 4,1 / 40,1 / 400$ prozentigen alkoholischen Terephtalaldehydlösungen ermittelt, indem mit jeder Lösung eine Serie von photographischen Aufnahmen mit in geometrischer Reihe ansteigenden Expositionszeiten auf eine Platte gemacht wurde. Die Figur zeigt die erhaltenen Kurven im Zeit-Wellenlängendiagramm. (Kurve II und III).

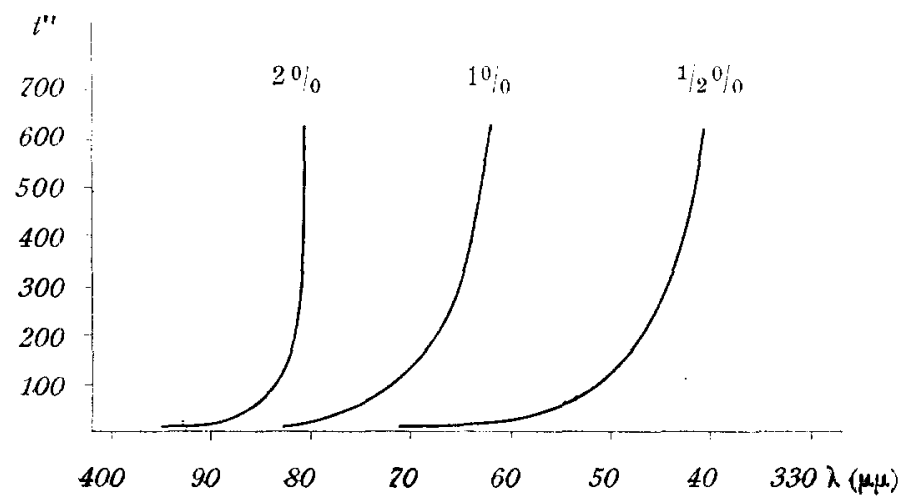

Kurve II. 


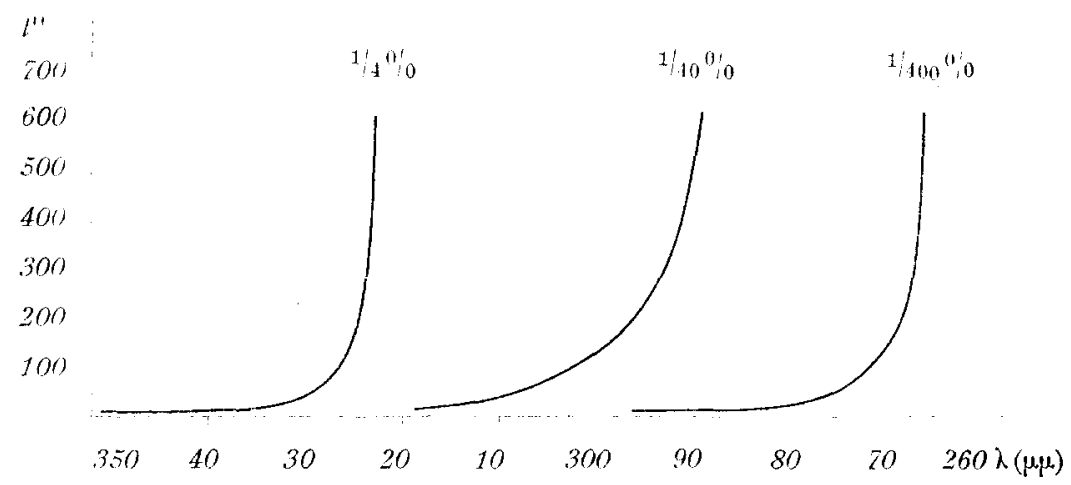

Kurve III.

Konstruiert man eine Kurve aus den Grenzwerten der Absorption für verschiedene Konzentrationen bei gleicher Expositionszeit, so erhält man ein ähnliches Bild über die Absorptionsverhältnisse, das bei Zugrundelegung des Beer'schen Gesetzes die Eindringungstiefe der einzelnen Strahlengattungen veranschaulicht.

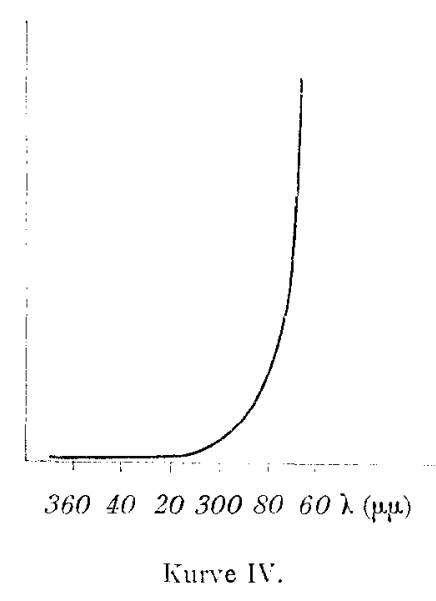

Eine weitere Begrenzung der Strahlengattung ist mit der Durchlässigkeit des verwendeten Eprouvettenglases gegeben. Bei der Prüfung eines Splitters auf seine absorbierende Wirkung nach den oben genannten Methoden zeigte es sich, daß die 
verwendete Glassorte in der gegebenen Dicke von $0.7 \mathrm{~mm}$ bis $300 \mu \mu$ abwärts aile Strahlen fast ungeschwächt durchläßt, an dieser Stelle aber ziemlich scharf abschneidet, daß die Absorption also erst für jene kurzwelligen Strablen beginnt, die das auffallende Sonnenlicht eben nicht mehr enthält, daß somit durch diese Glasschichte keine merkbare Beschränkung der Strahlenwirkung bei Verwendung von Sonnenlicht eintritt.

Zieht man nun die Emission der Quecksilberdampf-Quarzlampe in Vergleich, ${ }^{1}$ so zeigt sich die Tatsache, dali diese im Strahlenbezirk 370 bis 300 i $\mu$ nur ein engbegrenztes starkes Linienbündel ( $\wedge 3663$ bis 3650 ) und zwei schwächere Bündel bei 3133 bis 3126 und 3039 bis 3022 aufweist, von denen be Verwendung dieser Lichtquelle für die Reaktion die ganze Wirkung herrührt.

Ich gestatte mir an dieser Stelle Herrn Hofrat Prof. Dr J. M. Eder für das Entgegenkommen und die Unterstützung den wärmsten Dank auszusprechen.

Siehe Eaer und Valenta, "Atlas der typischen Metallspektren 